\title{
Motion Analysis and the Anterior Cruciate Ligament: Classification of Injury Risk
}

\author{
Nathaniel A. Bates, PhD ${ }^{1,2}$ Timothy E. Hewett, $\mathrm{PhD}^{1,2,3,4}$ \\ ${ }^{1}$ Department of Orthopedic Surgery, Mayo Clinic, Rochester, Minnesota \\ 2 Department of Sports Medicine, Sports Health and Performance \\ Institute, The Ohio State University, Columbus, Ohio \\ ${ }^{3}$ Department of Physical Medicine and Rehabilitation, Mayo Clinic, \\ Rochester, Minnesota \\ ${ }^{4}$ Department of Physiology and Biomedical Engineering, Mayo Clinic, \\ Rochester, Minnesota
}

J Knee Surg 2016;29:117-125.

\begin{abstract}
Address for correspondence Timothy E. Hewett, PhD, Department of Biomechanics, Mayo Clinic, 200 First St SW, R0_GU_01_28BIOM, Rochester, MN 55905 (e-mail: hewett.timothy@mayo.edu).
\end{abstract}

\author{
Abstract \\ Keywords \\ - anterior cruciate \\ ligament injury \\ - injury risk \\ classification \\ - motion capture \\ - motion analysis \\ - neuromuscular \\ training
}

Anterior cruciate ligament $(\mathrm{ACL})$ injuries are common, catastrophic events that incur large expense and lead to degradation of the knee. As such, various motion capture techniques have been applied to identify athletes who are at increased risk for suffering $\mathrm{ACL}$ injuries. The objective of this clinical commentary was to synthesize information related to how motion capture analyses contribute to the identification of risk factors that may predict relative injury risk within a population. Individuals employ both active and passive mechanisms to constrain knee joint articulation during motion. There is strong evidence to indicate that athletes who consistently classify as high-risk loaders during landing suffer from combined joint stability deficits in both the active and passive knee restraints. Implementation of prophylactic neuromuscular interventions and biofeedback can effectively compensate for some of the deficiencies that result from poor control of the active knee stabilizers and reduce the incidence of $A C L$ injuries.
More than $55 \%$ of all athletic injuries are incurred on the lower extremity, ${ }^{1-4}$ while damage specific to the knee accounts for approximately $15 \%$ of all athletic injuries. ${ }^{5}$ Overall, $43 \%$ of these knee injuries are classified as strains or sprains, which makes them the third most prevalent form of lower extremity injury with a rate of 102 incidents per 100,000 athletes per year. ${ }^{6}$ Of these knee injuries, it is estimated that $45 \%$ involve internal knee trauma, and $49 \%$ of those entail anterior cruciate ligament (ACL) rupture, ${ }^{7}$ as 1 in 3,000 persons are likely to suffer an ACL disruption each year. ${ }^{8}$ However, ACL injury is a sex-specific event, as females are 2 to 10 times more likely to suffer ACL disruption than their male counterparts, ${ }^{9-14}$ which produces an incidence rate of $1 \mathrm{ACL}$ tear in every 50 to 70 female athletes per year. ${ }^{15}$ These high incidence rates of ACL rupture lead to an estimated 250,000 ACL tears and 127,000 ACL reconstructions (ACLR) annually in the United States. ${ }^{16,17}$ With conservative repair estimates ranging from $\$ 5,000$ to $\$ 44,000$ per ACLR depending on the type of repair and

received

March 23, 2015

accepted after revision

May 26, 2015

published online

September 18, 2015 severity of injury, ${ }^{13,18-20}$ the annual medical expense of ACL injury treatments in the United States alone may exceed $\$ 2$ billion. Worldwide, it is estimated that the annual incidence of ACL tears could reach as high as 2 million patients, ${ }^{21}$ which would exponentially increase these costs. Unfortunately, despite the expense associated with ACLR, surgical repair has not been found to significantly reduce the longterm outlook of knee osteoarthritis compared with nonoperative rehabilitation. ${ }^{22,23}$ As many as $86 \%$ of patients demonstrated early onset osteoarthritis following ACLR and 75\% report degradation in knee quality of life within 20 years postsurgery. ${ }^{23-25}$ For these reasons, the focus on treating ACL injuries may be best served through identification and treatment of modifiable injury risk factors that may prevent ruptures before they happen.

Two- and three-dimensional (2D and 3D) motion analysis systems have been used in vivo to identify, classify, and associate biomechanical risk factors with the likelihood of
Copyright ( $\odot 2016$ by Thieme Medical Publishers, Inc., 333 Seventh Avenue, New York, NY 10001, USA. Tel: +1(212) 584-4662.
DOI http://dx.doi.org/ 10.1055/s-0035-1558855. ISSN 1538-8506. 


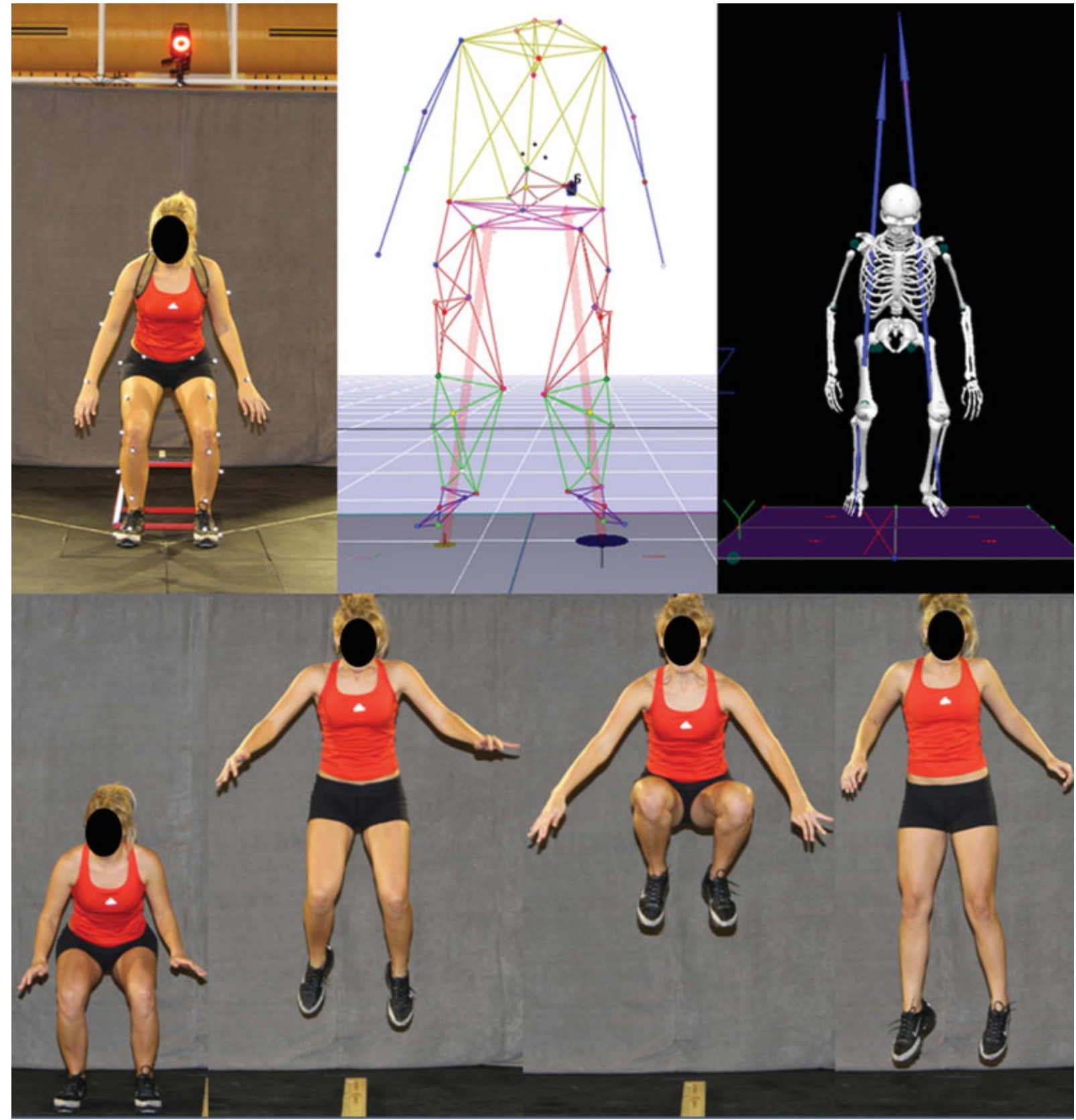

Fig. 1 (Top row) From left to right depicts three-dimensional (3D) motion capture models at the minimum center of gravity for a drop vertical jump performed in vivo within a 3D motion analysis laboratory, within the tracking software used to process 3D positional data from the in vivo markers, and within the musculoskeletal modeling software used to process 3D kinematic and kinetic biomechanics from the positional data and recorded ground reaction forces. Knee abduction torque from a drop vertical jump is commonly used to assess anterior cruciate ligament injury risk in 3D motion analyses. ${ }^{26,75}$ (Bottom row) Progression of a tuck jump task during two-dimensional (2D) motion analysis. The tuck jump task has been demonstrated as a clinician friendly, 2D assessment that can be used to identify high-risk mechanics and provide direction for neuromuscular intervention. ${ }^{44}$

future ACL injury within athletic populations (-Fig. 1). Specifically, in a cohort of 205 female athletes, 3D motion analysis prospectively determined that those who went on to $\mathrm{ACL}$ injury expressed larger knee abduction moments when landing from a drop vertical jump task than did healthy controls. ${ }^{26}$ This association between frontal plane knee torque and increased ligament loading, which was initially defined through motion analysis, has since been affirmed in a multitude of in vitro, in situ, and in sim models. ${ }^{27-29}$ In addition, 3D motion analysis has identified that decreased knee flexion, ${ }^{30}$ increased hip adduction, ${ }^{31-33}$ and greater trunk instability ${ }^{34-37}$ when landing from a jump are all related to abnormal loading at the knee and potentially increased ACL injury risk. Many of these specific factors identified in 3D models can be generalized in 2D motion analyses that are more cost-effective to the clinical environment. Relative presence of trunk instability, ${ }^{38,39}$ knee valgus angle, ${ }^{40}$ and knee flexion angle, ${ }^{40}$ and knee excursion in the 
frontal plane ${ }^{41}$ can be assessed in 2D capture. While not as precise as $3 \mathrm{D}$ analyses, these $2 \mathrm{D}$ generalizations have effectively been used to adapt biomechanical nommograms that identify athletes within an athletic cohort who are predisposed to ACL injury risk. ${ }^{40-44}$

As previously stated, the intent behind the identification of high-risk biomechanical behaviors and the athletes that display them is to treat and prevent ACL injuries before they occur. It has been repeatedly demonstrated that prophylactic neuromuscular interventions can have a positive influence on the reduction of ACL injuries within an athletic population. ${ }^{18,45-50}$ Neuromuscular training (NMT) is effective in reducing the magnitude of knee abduction moments generated by athletes during the performance of athletic tasks. ${ }^{51-54}$ As these frontal plane torques are directly associated with ACL injury, ${ }^{26}$ it is likely that decreasing their magnitudes is in part responsible for the overall reduction in injury incidence following NMT. Furthermore, NMT has been demonstrated to have a greater biomechanical effect on high-injury risk athletic populations than medium- or lowrisk cohorts. ${ }^{52}$ Accordingly, the classification of athletes in injury risk levels and definition of the underlying mechanisms that lead to these levels of risk may be vital to maximize the future efficacy of ACL injury prevention.

In this clinical prediction commentary, we synthesize information related to how motion capture analyses contribute to the identification of risk factors that may predict relative injury risk within a population. We argue that an athlete's relative ACL injury risk is dependent on which systems of control an athlete can effectively employ to restrain the knee joint during athletic tasks. In the first section of this commentary, we define the systems of control available at the knee and identify differences in mechanical outcomes between effective and less effective systems. In the second section, we identify the divisions of relative ACL injury risk based on knee abduction moment and justify our stated arguments. In subsequent sections, we address how robust the classifications of injury risk may be and examine the effectiveness of incorporation of biofeedback techniques with motion capture analysis to reduce relative injury risk within an athlete and an athletic population.

\section{Knee Joint Restraints}

Motion at the knee is constrained by a series of active and passive restraints that work in conjunction to stabilize the articulating structures when forces and perturbations are applied during an athletic maneuver. Active restraints reference the musculature surrounding the joint and the neuromuscular control mechanisms used to activate this musculature, specifically, the proprioceptive, kinesthetic, visual, vestibular, and motor command systems. ${ }^{55}$ With respect to the $A C L$, it has been hypothesized that ligament injury risk is related to measureable and modifiable deficits within these neuromuscular control mechanisms that influence muscle strength, power, and activation and, ultimately, knee joint and ACL loads. ${ }^{26,56-58}$ Relative to muscle mechanics, quadriceps activation has traditionally been seen as antagonist to the ACL, adding strain, whereas, hamstrings activation has been agonist, providing protection. ${ }^{59}$ Accordingly, a poor quadriceps-hamstrings activation ratio can lead to increased knee extension during landing and excess anterior tibial translation, both of which increase ACL strain. ${ }^{60-62}$ This preferential activation of knee extensors over knee flexors to stabilize the knee during motion tasks is termed quad dominance. ${ }^{63}$ Quadriceps-dominant traits can lead to lower flexion angles upon landing that indicate less time and movement to absorb the impulse forces generated from landing and lead to greater joint force generation as a result of this more extended position. ${ }^{55,62,64}$ A second deficit within active knee restraints is leg dominance, where the imbalance between muscular strength and recruitment on opposing limbs lead to contralateral asymmetries. ${ }^{63}$ Kinetic and kinematic analyses have shown this type of deficit to be especially prevalent in female athletes, as they demonstrate greater limb asymmetries, especially in regard to frontal plane kinematics, than their male counterparts. ${ }^{57,65}$ Furthermore, motion analysis investigations have revealed that contralateral asymmetries are greater following ACLR; and therefore, may be related to the increased likelihood of secondary or contralateral ACL rupture that exists within the ACL-injured population following return to sport. ${ }^{66}$ The common outcome brought on by the variety of neuromuscular control deficits documented here is that the active knee stabilizers are unable to restrain the joint from articulating into dynamic valgus rapid deceleration tasks, a trait that is able predict ACL injury risk with $78 \%$ sensitivity and $73 \%$ specificity. ${ }^{26}$

Passive knee restraints reference the ligaments, bony structures, and generalized laxity within the joint that contribute to the mechanical constraint of motion in the absence of a neuromuscular response. Excessive employment of the passive restraints in the knee can be related to insufficient control of the active restraints that results in a ligament dominant condition. ${ }^{63,67}$ Ligament dominance refers to decreased medial/lateral muscle control that leads to high valgus torque and vertical ground reaction forces. ${ }^{63,66,68,69}$ In this condition, the ground reaction forces dictate joint movement direction rather than the musculature and allow for substantial loading of the ligamentous structures within the knee. ${ }^{63,67}$ In addition, increased knee laxity, especially in the anteroposterior degree of freedom, leads to increased tibial translation, which leads to additional loading of the knee ligaments as they mechanically work to resist this excess motion. Morphologic changes that can relate to ACL injury risk include tibial plateau slope ${ }^{70}$ and femoral notch width. ${ }^{71}$ As posterior tibial slope increases, the femur is more likely to slide posteriorly on the tibial plateau, which places mechanical demand on the ACL as the ligament resists up to $85 \%$ of anterior tibial translational force in the knee. ${ }^{70,72,73}$ As the femoral notch width decreases, the likelihood of ACL impingement increases, which adds strain to the ligament. ${ }^{74}$ Unlike active restraints, these passive knee restraints cannot be altered by intervention training and are thus referred to as nonmodifiable risk factors as changes require invasive surgical intervention. 


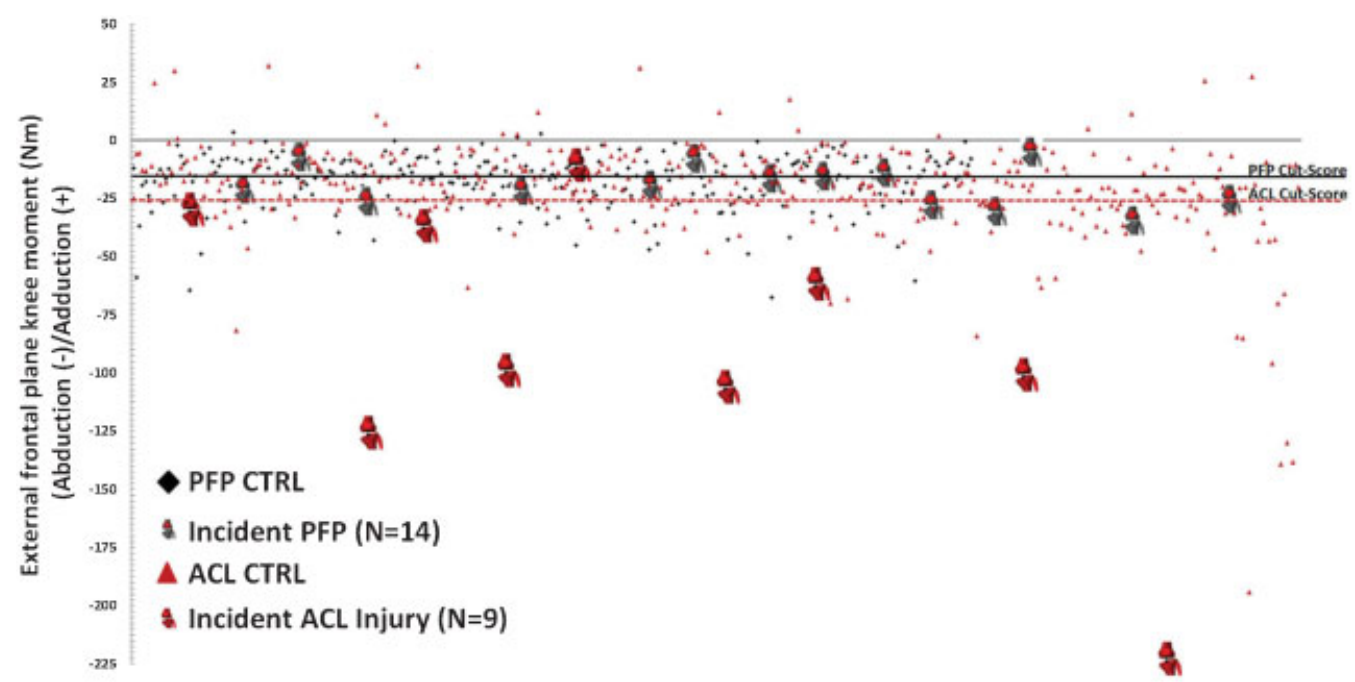

Fig. 2 Peak knee abduction moments generated by two prospectively analyzed cohorts of adolescent female athletes during a DVJ task. The first cohort (red) was used to determine the magnitude of knee abduction torque that would predict ACL injury risk, ${ }^{26}$ while the second cohort (black) was used to determine the magnitude of knee abduction torque that would predict patellofemoral pain risk. ${ }^{75,77,78}$ The horizontal lines designate relative injury risk divisions for the medium (solid line) and high (dashed line) risk groups. (Reprinted with permission from Myer et al ${ }^{75}$ ). $\mathrm{ACL}$ anterior cruciate ligament; CTRL, Control; PFP, patellofemoral pain.

\section{Relative Injury Risk}

Traditionally, the relative level of ACL injury risk displayed by an athlete has been determined by the magnitude of knee abduction moment he or she generates during drop landing (-Fig. 2). ${ }^{26,40}$ In a cohort of 205 female athletes who underwent 3D motion analysis and were prospectively monitored for subsequent ACL injury, it was discovered that those athletes who went on to injury exhibited greater knee abduction moments than did healthy controls. ${ }^{26}$ The mean peak knee abduction torque for injury patients was 21.74 $\mathrm{N}^{*} \mathrm{~m}$. Within this population, it was later assessed that peak knee abduction moments above $25.3 \mathrm{~N}^{*} \mathrm{~m}$ increased risk for subsequent ACL injury from 0.4 to $6.8 \%$; thus, this was established as the cutoff threshold for the classification of high injury risk athletes. ${ }^{75}$ Similarly, during 3D motion analysis of the same drop vertical jump task, a separate patient population with patellofemoral pain was found to generate greater peak knee abduction moments on their uninvolved limb than did healthy controls. ${ }^{76}$ Within these two population cohorts that were examined separately for ACL injury and patellofemoral pain, the incidence of patellofemoral pain was found to be 2.2 times greater than ACL injury when normalized for athlete seasons. ${ }^{75}$ This discrepancy in incidence indicated that a tertiary level of injury risk may be present. Indeed, a second investigation based on the same 3D motion analysis techniques that defined high injury risk athletes found that probability of patellofemoral pain occurrence increased dramatically in athletes with peak knee abduction moments greater than 15.4 $\mathrm{N}^{*} \mathrm{~m} .{ }^{75,77}$ This threshold predicted knee abduction load associated with increased patellofemoral pain risk with $92 \%$ sensitivity and $74 \%$ specificity and, as such, was determined to represent a medium injury risk classification among athletes. ${ }^{75,77}$
As noted previously, the data from these 3D motion analyses were disseminated into algorithms that could predict both ACL injury ${ }^{40-42,78}$ and patellofemoral pain. ${ }^{77}$ The purpose of these 2D injury risk nommograms was to create clinically relevant tools that could quickly, accurately, and cost-efficiently categorize athletes into relative injury risk groups based on these peak knee abduction moment divisions. While 3D motion analysis is an effective laboratory tool, these systems are cost prohibitive and involve intensive data analysis, which makes them inaccessible to most clinical environments. ${ }^{40,79} \mathrm{To}$ this effect, the selected clinically based nomograms involve minimal investment (two video cameras and laptop), ${ }^{40,79}$ can be quickly and reliably assessed by a trained clinician, ${ }^{80}$ and exhibit high sensitivity and moderate specificity in the classification of relative risk for ACL injury (84 and 67\%, respectively) or patellofemoral pain (92 and $46 \%$, respectively). ${ }^{77,81}$

Despite having equivalent fall heights, 3D motion capture analysis of the first and second landings of a drop vertical jump (DVJ) has revealed several differences in kinetic and kinematic performance. ${ }^{82}$ Because of these differences, the first landing may be more predisposed to exhibit larger frontal plane moments than the second. As such the two landing phases exhibit a potential shift in injury risk classification. A population of 239 adolescent female athletes underwent 3D motion analysis as they completed both the first and second landings of a DVJ, as was described previously in the literature. ${ }^{82-84}$ of these athletes, both landings were successfully captured on 206 subjects. Peak knee abduction moments calculated from the motion capture model were used to classify these athletes into relative injury risk groups as specified earlier. It was found that during the first landing $57.2 \%$ were classified as low ACL injury risk, $21.8 \%$ were medium risk, and $20.9 \%$ were high risk; while during the second landing $66.5 \%$ were low risk, $21.4 \%$ were medium risk, and $12.1 \%$ were high risk ( - Fig. 3 ). This cohort of athletes was 


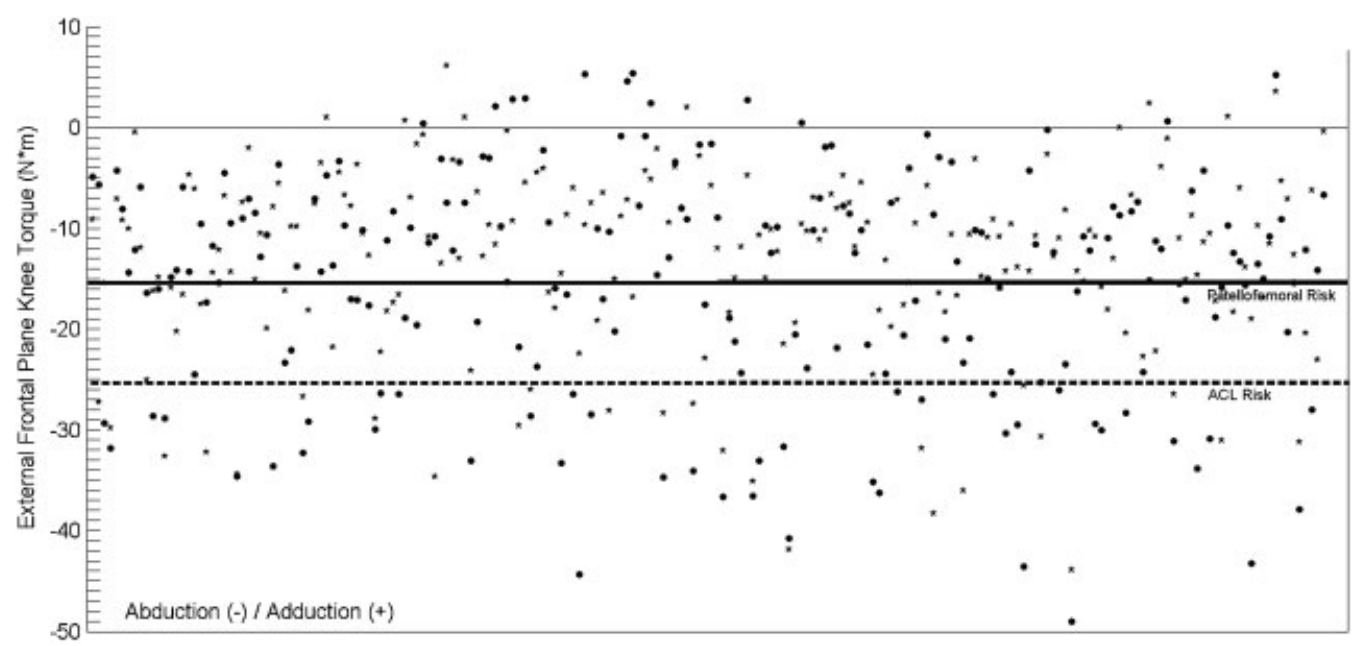

Fig. 3 Depiction of risk category divisions during the first (circles) and second (stars) landing of a DVJ within the 206 adolescent female athletes examined in this article. Peak abduction torques below the patellofemoral risk line designate the medium injury risk classification, while values below the anterior cruciate ligament risk line designate the high injury risk classification.

previously shown to generate a slightly larger mean peak frontal plane knee torque during the first landing of a drop vertical jump than during the second ${ }^{82}$ The frequency of highrisk classifications between landings presented here corresponded with that finding; however, the relative infrequency of high-risk kinetics during the second landing may indicate that it is more selective in diagnosis. Of the athletes who expressed high-risk knee abduction torque during the first landing, 37.2\% continued to demonstrate torque magnitudes indicative of high-risk biomechanics during the second landing, while 30.2 and $32.6 \%$ exhibited medium and low risk, respectively (-Fig. 4). Conversely, $64.0 \%$ of athletes who exhibited "high-risk" torques in the second landing were also classified as high risk in the first landing. Kinematic and kinetic variability within the performance of an athletic task, as captured by motion analysis systems, has been previously documented. ${ }^{80,85}$ For peak knee abduction moments within a session, the interclass correlation coefficient was 0.931 , which represents excellent reliability and consistent results. ${ }^{85}$ Accordingly, it would be expected that subjects would steadily demonstrate the same level of ACL injury risk when landing from a jump. However, the classification consistency of this data did not match the reported reliability. It is possible to generate high-risk knee abduction torques with poor control over either the dynamic or passive knee restraints, but perhaps athletes who consistently exhibit high-risk frontal plane torques between landings represent subjects who express poor control over both mechanisms of knee restraint.

The authors propose that athletes who continually express relatively high levels of injury risk during rapid deceleration tasks have poor control over both their active and passive mechanisms of knee restraint. Those athletes who express medium injury risk, or fluctuate between groups, may only express poor control over one set of knee restraints, either the active or passive systems. During the performance of athletic tasks, athletes generate large impulse forces at the knee that

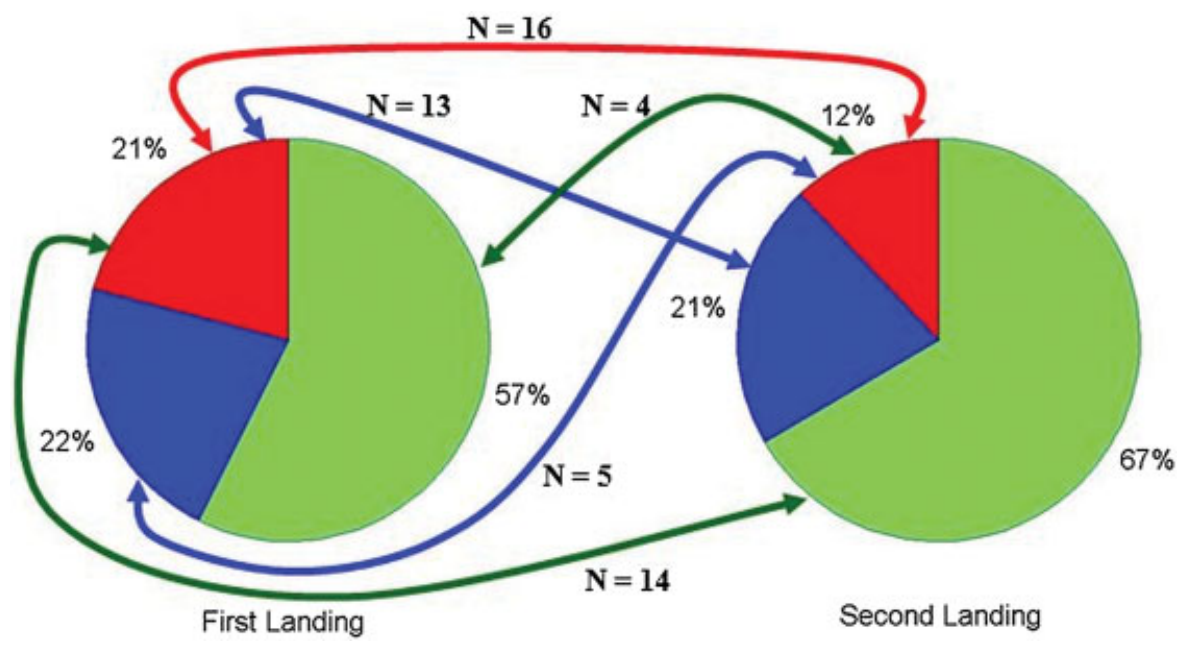

Fig. 4 Pie chart depiction of anterior cruciate ligament injury risk classifications for 206 adolescent female athletes during the first and second landing of a DVJ. Lines demonstrate the number of high-risk athletes that changed injury risk classifications between landings. Only 16 athletes (7.8\%) were classified as high risk in both landings based on their peak knee abduction moment. 
are primarily absorbed over time as the musculature flexes the joint through a range of motion. ${ }^{30,55}$ When muscular strength or activation proves insufficient to properly flex the joint and restrain these impulse forces, the knee is forced into frontal and transverse plane rotations. ${ }^{57,86}$ As the musculature at the knee primarily functions in the sagittal plane, such perturbations may require passive structures to restrain the joint from collapse. ${ }^{57,86-88}$ Therefore, as relative injury risk levels are classified by peak knee abduction torque generated during landing, ${ }^{26}$ athletes who exhibit high knee abduction torques during drop vertical jumps have, by definition, identified themselves to have poor neuromuscular control. This is because their musculature failed to constrain the resultant ground reaction forces within the sagittal plane of the knee. ${ }^{57,86}$ However, not all athletes who express weak musculature or poor hamstrings to quadriceps strength ratios generate high knee abduction moments during landing.

The previously described 206 athletes who were successfully evaluated via 3D motion analysis during the first and second landing of a drop vertical jump were also assessed for hamstrings and quadriceps strength on a dynamometer using previously published methods. ${ }^{89}$ of 206 athletes, 117 were found to have poor hamstrings-to-quadriceps strength ratios (below 0.60 hamstrings-to-quadriceps peak strength at 300 degree/second $)^{90}$; yet, within this subset, only 29 and 15 of these athletes generated knee abduction torques greater than $25.3 \mathrm{~N}^{*} \mathrm{~m}$ in the first and second landing, respectively. Furthermore, as landing from a drop vertical jump generates ground reaction force magnitudes up to $4.5 \times$ bodyweight, ${ }^{84}$ if active knee restraints were the sole contributor to injury risk classification, athletes with the lowest peak strength to body mass ratio would be expected to almost entirely comprise the high injury risk group. However, of the 43 athletes classified as high risk in the first landing, only 17 came from athletes who were in the bottom half of strength to body mass ratios. This behavior indicated that well-developed passive restraints within the knee may be able to compensate for active restraint deficiencies. Furthermore, this implicated that consistent high-risk torque generation may require poor control in both active and passive knee restraint mechanisms. In addition to motion capture and strength measures, the same cohort of adolescent female athletes underwent arthrometry evaluations for joint laxity using previously published methods. ${ }^{91}$ Again, increased knee laxity alone was not sufficient to predict knee injury risk as only 21 of 43 high-risk subjects exhibited relatively high knee laxity values (greater than $10.22 \mathrm{~mm}$ anterior translation under $134 \mathrm{~N}$ of anterior force as this was the mean laxity value reported in the contralateral limbs of patients with ACL injury). ${ }^{87,88}$ of the 206 subjects, 43 exhibited each poor hamstrings-to-quadriceps ratio, lower peak-strength-tobody-mass ratio, and high joint laxity. ${ }^{91,92}$ Between both landings, 11 of $43(25.6 \%)$ subjects in this subset also exhibited high knee abduction moments during landing. While, far from precise in their indication of risk, the presence of high peak knee abduction torques in these athletes with poor active and passive knee restraints was greater than it was in the overall cohort. These data support that underdeveloped passive restraints contribute to overall injury risk and that a consistent high injury risk classification may be indicative of poor coordination over both systems of knee stabilization.

\section{Neuromuscular Interventions}

Prophylactic neuromuscular interventions have been shown to effectively reduce ACL injury incidence over time through successful alteration of negative biomechanical tendencies that contribute to injury risk. ${ }^{18,45-50}$ Specifically, a review of interventions discovered that NMT produces a relative risk reduction of $73.4 \%$ for noncontact ACL injury in female athletes and that these interventions prevent approximately one injury per every 108 individuals that participate in training. ${ }^{47}$ It was also found that those neuromuscular interventions that incorporated multiple types of training, each strengthening, plyometric, and balance exercises, were more effective in the reduction of injury rates than interventions focused on a single training type. ${ }^{47}$ An additional mechanism investigators have recently begun to incorporate into intervention training is biofeedback. Biofeedback utilizes motion analysis techniques to assess deficiencies in dynamic task performance and immediately reinforces them with visual or audible cues that indicate directly to the patient how he or she might optimize movement patterns. In clinical settings, biofeedback has primarily been used to treat gait abnormalities in both pediatric and adult populations. ${ }^{93}$ Furthermore, investigation has found that biofeedback can instantly reduce relative injury risk for those athletes that exhibit high-risk mechanics. ${ }^{52}$ Motion analysis investigations have demonstrated that feedback will correct for pain and deficits in gait with moderate-to-large treatment effect on adult patients. ${ }^{93}$ During jump landing, feedback has been shown to effectively reduce jump landing forces, ${ }^{94}$ reduce trunk sway, ${ }^{95}$ and reduce knee hyperextension. ${ }^{96}$ Furthermore, real-time kinetic-focused biofeedback implemented in conjunction with 3D motion analysis during a squat activity has been found to immediately reduce peak knee abduction moment during a drop vertical jump, the primary indicator of ACL injury risk, by $32.8 \%$ in adolescent female athletes. ${ }^{97}$ As previously noted, increased magnitudes of each of these factors has been associated with ACL injury risk; therefore, reduction through biofeedback exemplifies the potential of this tool to further limit the incidence of ACL rupture. Future implementations of biofeedback could be applied in conjunction with the previously documented injury risk nomograms based on 2D motion analysis as a clinically relevant mechanism for the diagnosis and immediate initiation of corrective treatment for athletes who exhibit high-risk tendencies.

\section{Summary}

Motion-capture analyses have made significant contributions to the identification of factors that contribute to ACL injury risk. The classification of relative injury risk via $2 \mathrm{D}$ and $3 \mathrm{D}$ motion analysis techniques plays an essential role for both researchers and clinicians who wish to intervene and prevent ACL in athletic populations. There is strong evidence to 
indicate that athletes who consistently classify as high-risk loaders during landing suffer from combined joint stability deficits in both their active and passive restraints at the knee joint. Implementation of prophylactic neuromuscular interventions has been shown to effectively compensate for some of the deficiencies that results from poor control of the active knee stabilizers and, in turn, reduce the incidence of ACL injuries. With continued development of these interventions, such as through the incorporation of motion analysis-based biofeedback, researchers, and clinicians can continue to improve the efficacy of ACL injury prevention initiatives.

\section{References}

1 Agel J, Olson DE, Dick R, Arendt EA, Marshall SW, Sikka RS. Descriptive epidemiology of collegiate women's basketball injuries: National Collegiate Athletic Association Injury Surveillance System, 1988-1989 through 2003-2004. J Athl Train 2007;42(2): 202-210

2 Dick R, Ferrara MS, Agel J, et al. Descriptive epidemiology of collegiate men's football injuries: National Collegiate Athletic Association Injury Surveillance System, 1988-1989 through 2003-2004. J Athl Train 2007;42(2):221-233

3 Dick R, Hertel J, Agel J, Grossman J, Marshall SW. Descriptive epidemiology of collegiate men's basketball injuries: National Collegiate Athletic Association Injury Surveillance System, 19881989 through 2003-2004. J Athl Train 2007;42(2):194-201

4 Dick R, Putukian M, Agel J, Evans TA, Marshall SW. Descriptive epidemiology of collegiate women's soccer injuries: National Collegiate Athletic Association Injury Surveillance System, 19881989 through 2002-2003. J Athl Train 2007;42(2):278-285

5 Yard E, Comstock D. Injury patterns by body mass index in US high school athletes. J Phys Act Health 2011;8(2):182-191

6 Lambers K, Ootes D, Ring D. Incidence of patients with lower extremity injuries presenting to US emergency departments by anatomic region, disease category, and age. Clin Orthop Relat Res 2012;470(1):284-290

7 Majewski M, Susanne H, Klaus S. Epidemiology of athletic knee injuries: A 10-year study. Knee 2006;13(3):184-188

8 Miyasaka KC, Daniel DM, Stone ML, Hirshman P. The incidence of knee ligament injuries in the general population. Am J Knee Surg $1991 ; 4(1): 3-8$

9 Arendt EA, Agel J, Dick R. Anterior cruciate ligament injury patterns among collegiate men and women. J Athl Train 1999; 34(2):86-92

10 Boden BP, Dean GS, Feagin JA Jr, Garrett WE Jr. Mechanisms of anterior cruciate ligament injury. Orthopedics 2000;23(6): 573-578

11 Toth AP, Cordasco FA. Anterior cruciate ligament injuries in the female athlete. J Gend Specif Med 2001;4(4):25-34

12 Hootman JM, Dick R, Agel J. Epidemiology of collegiate injuries for 15 sports: summary and recommendations for injury prevention initiatives. J Athl Train 2007;42(2):311-319

13 Gianotti SM, Marshall SW, Hume PA, Bunt L. Incidence of anterior cruciate ligament injury and other knee ligament injuries: a national population-based study. J Sci Med Sport 2009;12(6): 622-627

14 Griffin LY, Agel J, Albohm MJ, et al. Noncontact anterior cruciate ligament injuries: risk factors and prevention strategies. J Am Acad Orthop Surg 2000;8(3):141-150

15 Myer GD, Ford KR, Hewett TE. Preventing ACL injuries in Women. J Musculoskel Med. 2006;23(1):12-38

16 Johnson DL, Warner JJP. Diagnosis for anterior cruciate ligament surgery. Clin Sports Med 1993;12(4):671-684
17 Kim S, Bosque J, Meehan JP, Jamali A, Marder R. Increase in outpatient knee arthroscopy in the United States: a comparison of National Surveys of Ambulatory Surgery, 1996 and 2006. J Bone Joint Surg Am 2011;93(11):994-1000

18 Hewett TE, Lindenfeld TN, Riccobene JV, Noyes FR. The effect of neuromuscular training on the incidence of knee injury in female athletes. A prospective study. Am J Sports Med 1999;27(6): 699-706

19 Bates NA, McPherson AL, Rao MB, Myer GD, Hewett TE. Characteristics of inpatient anterior cruciate ligament reconstructions and concomitant injuries (e-pub ahead of print). Knee Surg Sports Traumatol Arthrosc 2014; doi: 10.1007/s00167-014-3478-3

20 Nagda SH, Altobelli GG, Bowdry KA, Brewster CE, Lombardo SJ. Cost analysis of outpatient anterior cruciate ligament reconstruction: autograft versus allograft. Clin Orthop Relat Res 2010;468(5): 1418-1422

21 Renström PA. Eight clinical conundrums relating to anterior cruciate ligament (ACL) injury in sport: recent evidence and a personal reflection. Br J Sports Med 2013;47(6):367-372

22 Neuman P, Englund M, Kostogiannis I, Fridén T, Roos H, Dahlberg LE. Prevalence of tibiofemoral osteoarthritis 15 years after nonoperative treatment of anterior cruciate ligament injury: a prospective cohort study. Am J Sports Med 2008;36(9):1717-1725

23 Myklebust G, Bahr R. Return to play guidelines after anterior cruciate ligament surgery. Br J Sports Med 2005;39(3):127-131

24 von Porat A, Roos EM, Roos H. High prevalence of osteoarthritis 14 years after an anterior cruciate ligament tear in male soccer players: a study of radiographic and patient relevant outcomes. Ann Rheum Dis 2004;63(3):269-273

25 Lohmander LS, Ostenberg A, Englund M, Roos H. High prevalence of knee osteoarthritis, pain, and functional limitations in female soccer players twelve years after anterior cruciate ligament injury. Arthritis Rheum 2004;50(10):3145-3152

26 Hewett TE, Myer GD, Ford KR, et al. Biomechanical measures of neuromuscular control and valgus loading of the knee predict anterior cruciate ligament injury risk in female athletes: a prospective study. Am J Sports Med 2005;33(4):492-501

27 Levine JW, Kiapour AM, Quatman CE, et al. Clinically relevant injury patterns after an anterior cruciate ligament injury provide insight into injury mechanisms. Am J Sports Med 2013;41(2):385-395

28 Withrow TJ, Huston LJ, Wojtys EM, Ashton-Miller JA. The effect of an impulsive knee valgus moment on in vitro relative ACL strain during a simulated jump landing. Clin Biomech (Bristol, Avon) 2006;21(9):977-983

29 Shin CS, Chaudhari AM, Andriacchi TP. The effect of isolated valgus moments on ACL strain during single-leg landing: a simulation study. J Biomech 2009;42(3):280-285

30 Dufek JS, Bates BT. Biomechanical factors associated with injury during landing in jump sports. Sports Med 1991;12(5):326-337

31 Myer GD, Imwalle LE, Ford KR, Hewett TE. Hip adduction, not hip internal rotation, dictates knee motion related to acl injury during cutting maneuvers. Med Sci Sports Exerc 2006;38(5):614-619

32 Imwalle LE, Myer GD, Ford KR, Hewett TE. Relationship between hip and knee kinematics in athletic women during cutting maneuvers: a possible link to noncontact anterior cruciate ligament injury and prevention. J Strength Cond Res 2009;23(8):2223-2230

33 Hewett TE, Ford KR, Myer GD, Wanstrath K, Scheper M. Gender differences in hip adduction motion and torque during a single-leg agility maneuver. J Orthop Res 2006;24(3):416-421

34 Hewett TE, Myer GD. The mechanistic connection between the trunk, hip, knee, and anterior cruciate ligament injury. Exerc Sport Sci Rev 2011;39(4):161-166

35 Winter DA. Biomechanics and Motor Control of Human Movement. 3rd ed. ed. New York, NY: John Wiley \& Sons, Inc.; 2005

36 Hewett TE, Torg JS, Boden BP. Video analysis of trunk and knee motion during non-contact anterior cruciate ligament injury in female athletes: lateral trunk and knee abduction motion are 
combined components of the injury mechanism. Br J Sports Med 2009;43(6):417-422

37 Zazulak BT, Hewett TE, Reeves NP, Goldberg B, Cholewicki J. The effects of core proprioception on knee injury: a prospective biomechanical-epidemiological study. Am J Sports Med 2007; 35(3):368-373

38 DiCesare CA, Bates NA, Myer GD, Hewett TE. The validity of 2dimensional measurement of trunk angle during dynamic tasks. Int J Sports Phys Ther 2014;9(4):420-427

39 Dingenen B, Malfait B, Vanrenterghem J, Robinson MA, Verschueren SM, Staes FF. Can two-dimensional measured peak sagittal plane excursions during drop vertical jumps help identify threedimensional measured joint moments? Knee 2015;22(2):73-79

40 Myer GD, Ford KR, Khoury J, Succop P, Hewett TE. Development and validation of a clinic-based prediction tool to identify female athletes at high risk for anterior cruciate ligament injury. Am J Sports Med 2010;38(10):2025-2033

41 Myer GD, Ford KR, Khoury J, Succop P, Hewett TE. Clinical correlates to laboratory measures for use in non-contact anterior cruciate ligament injury risk prediction algorithm. Clin Biomech (Bristol, Avon) 2010;25(7):693-699

42 Myer GD, Ford KR, Khoury J, Hewett TE. Three-dimensional motion analysis validation of a clinic-based nomogram designed to identify high ACL injury risk in female athletes. Phys Sportsmed 2011;39(1):19-28

43 Zebis MK, Andersen LL, Bencke J, Kjaer M, Aagaard P. Identification of athletes at future risk of anterior cruciate ligament ruptures by neuromuscular screening. Am J Sports Med 2009;37(10): 1967-1973

44 Myer GD, Ford KR, Hewett TE. Tuck Jump Assessment for Reducing Anterior Cruciate Ligament Injury Risk. Athl Ther Today 2008; 13(5):39-44

45 Myklebust G, Engebretsen L, Braekken IH, Skjølberg A, Olsen OE, Bahr R. Prevention of anterior cruciate ligament injuries in female team handball players: a prospective intervention study over three seasons. Clin J Sport Med 2003;13(2):71-78

46 Mandelbaum BR, Silvers HJ, Watanabe DS, et al. Effectiveness of a neuromuscular and proprioceptive training program in preventing anterior cruciate ligament injuries in female athletes: 2-year follow-up. Am J Sports Med 2005;33(7):1003-1010

47 Sugimoto D, Myer GD, McKeon JM, Hewett TE. Evaluation of the effectiveness of neuromuscular training to reduce anterior cruciate ligament injury in female athletes: a critical review of relative risk reduction and numbers-needed-to-treat analyses. $\mathrm{Br} J$ Sports Med 2012;46(14):979-988

48 Chappell JD, Limpisvasti O. Effect of a neuromuscular training program on the kinetics and kinematics of jumping tasks. Am J Sports Med 2008;36(6):1081-1086

49 Hewett TE, Myer GD, Ford KR. Reducing knee and anterior cruciate ligament injuries among female athletes: a systematic review of neuromuscular training interventions. J Knee Surg 2005;18(1): $82-88$

50 Hewett TE, Ford KR, Myer GD. Anterior cruciate ligament injuries in female athletes: Part 2, a meta-analysis of neuromuscular interventions aimed at injury prevention. Am J Sports Med 2006;34(3):490-498

51 Myer GD, Ford KR, McLean SG, Hewett TE. The effects of plyometric versus dynamic stabilization and balance training on lower extremity biomechanics. Am J Sports Med 2006;34(3): 445-455

52 Myer GD, Ford KR, Brent JL, Hewett TE. Differential neuromuscular training effects on ACL injury risk factors in"high-risk" versus "low-risk" athletes. BMC Musculoskelet Disord 2007;8(39):39

53 Myer GD, Ford KR, Brent JL, Hewett TE. The effects of plyometric vs. dynamic stabilization and balance training on power, balance, and landing force in female athletes. J Strength Cond Res 2006;20(2): 345-353
54 Myer GD, Ford KR, Palumbo JP, Hewett TE. Neuromuscular training improves performance and lower-extremity biomechanics in female athletes. J Strength Cond Res 2005;19(1):51-60

55 Lephart SM, Abt JP, Ferris CM. Neuromuscular contributions to anterior cruciate ligament injuries in females. Curr Opin Rheumatol 2002;14(2):168-173

56 Myer GD, Brent JL, Ford KR, Hewett TE. Real-time assessment and neuromuscular training feedback techniques to prevent ACL injury in female athletes. Strength Condit J 2011;33(3):21-35

57 Ford KR, Myer GD, Hewett TE. Valgus knee motion during landing in high school female and male basketball players. Med Sci Sports Exerc 2003;35(10):1745-1750

58 Myer GD, Ford KR, Hewett TE. Rationale and Clinical Techniques for Anterior Cruciate Ligament Injury Prevention Among Female Athletes. J Athl Train 2004;39(4):352-364

59 Renström P, Arms SW, Stanwyck TS, Johnson RJ, Pope MH. Strain within the anterior cruciate ligament during hamstring and quadriceps activity. Am J Sports Med 1986;14(1):83-87

60 Podraza JT, White SC. Effect of knee flexion angle on ground reaction forces, knee moments and muscle co-contraction during an impact-like deceleration landing: implications for the noncontact mechanism of ACL injury. Knee 2010;17(4):291-295

61 Bates NA, Myer GD, Shearn JT, Hewett TE. Anterior cruciate ligament biomechanics during robotic and mechanical simulations of physiologic and clinical motion tasks: a systematic review and meta-analysis. Clin Biomech (Bristol, Avon) 2015;30(1):1-13

62 Malinzak RA, Colby SM, Kirkendall DT, Yu B, Garrett WE. A comparison of knee joint motion patterns between men and women in selected athletic tasks. Clin Biomech (Bristol, Avon) 2001;16(5):438-445

63 Hewett TE, Paterno MV, Myer GD. Strategies for enhancing proprioception and neuromuscular control of the knee. Clin Orthop Relat Res 2002;(402):76-94

64 Colby S, Francisco A, Yu B, Kirkendall D, Finch M, Garrett W Jr. Electromyographic and kinematic analysis of cutting maneuvers. Implications for anterior cruciate ligament injury. Am J Sports Med 2000;28(2):234-240

65 Pappas E, Carpes FP. Lower extremity kinematic asymmetry in male and female athletes performing jump-landing tasks. J Sci Med Sport 2012;15(1):87-92

66 Paterno MV, Schmitt LC, Ford KR, et al. Biomechanical measures during landing and postural stability predict second anterior cruciate ligament injury after anterior cruciate ligament reconstruction and return to sport. Am J Sports Med 2010;38(10): 1968-1978

67 Hewett TE, Myer GD, Ford KR. The influence of growth and pubertal maturation on neuromuscular performance in highrisk female athletes. Med Sci Sports Exerc 2002;34(5):S247

68 Pappas E, Zampeli F, Xergia SA, Georgoulis AD. Lessons learned from the last 20 years of ACL-related in vivo-biomechanics research of the knee joint. Knee Surg Sports Traumatol Arthrosc 2013;21(4):755-766

69 Hewett TE, Ford KR, Hoogenboom BJ, Myer GD. Understanding and preventing acl injuries: current biomechanical and epidemiologic considerations - update 2010. N Am J Sports Phys Ther 2010;5(4): 234-251

70 Wordeman SC, Quatman CE, Kaeding CC, Hewett TE. In vivo evidence for tibial plateau slope as a risk factor for anterior cruciate ligament injury: a systematic review and meta-analysis. Am J Sports Med 2012;40(7):1673-1681

71 Alentorn-Geli E, Mendiguchía J, Samuelsson K, et al. Prevention of anterior cruciate ligament injuries in sports. Part I: systematic review of risk factors in male athletes. Knee Surg Sports Traumatol Arthrosc 2014;22(1):3-15

72 Butler DL, Noyes FR, Grood ES. Ligamentous restraints to anteriorposterior drawer in the human knee. A biomechanical study. J Bone Joint Surg Am 1980;62(2):259-270 
73 Markolf KL, Gorek JF, Kabo JM, Shapiro MS. Direct measurement of resultant forces in the anterior cruciate ligament. An in vitro study performed with a new experimental technique. J Bone Joint Surg Am 1990;72(4):557-567

74 Souryal TO, Freeman TR. Intercondylar notch size and anterior cruciate ligament injuries in athletes. A prospective study. Am J Sports Med 1993;21(4):535-539

75 Myer GD, Ford KR, Di Stasi SL, Foss KD, Micheli LJ, Hewett TE. High knee abduction moments are common risk factors for patellofemoral pain (PFP) and anterior cruciate ligament (ACL) injury in girls: is PFP itself a predictor for subsequent ACL injury? Br J Sports Med 2015;49(2):118-122

76 Myer GD, Ford KR, Barber Foss KD, et al. The incidence and potential pathomechanics of patellofemoral pain in female athletes. Clin Biomech (Bristol, Avon) 2010;25(7):700-707

77 Myer GD, Ford KR, Foss KD, Rauh MJ, Paterno MV, Hewett TE. A predictive model to estimate knee-abduction moment: implications for development of a clinically applicable patellofemoral pain screening tool in female athletes. J Athl Train 2014;49(3):389-398

78 Myer GD, Ford KR, Khoury J, Succop P, Hewett TE. Biomechanics laboratory-based prediction algorithm to identify female athletes with high knee loads that increase risk of ACL injury. Br J Sports Med 2011;45(4):245-252

79 Myer GD, Ford KR, Hewett TE. New method to identify athletes at high risk of ACL injury using clinic-based measurements and freeware computer analysis. Br J Sports Med 2011;45(4):238-244

80 Myer GD, Wordeman SC, Sugimoto D, et al. Consistency of clinical biomechanical measures between three different institutions: implications for multi-center biomechanical and epidemiological research. Int J Sports Phys Ther 2014;9(3):289-301

81 Ford KR, Myer GD, Hewett TE. Longitudinal effects of maturation on lower extremity joint stiffness in adolescent athletes. Am J Sports Med 2010;38(9):1829-1837

82 Bates NA, Ford KR, Myer GD, Hewett TE. Kinetic and kinematic differences between first and second landings of a drop vertical jump task: implications for injury risk assessments. Clin Biomech (Bristol, Avon) 2013;28(4):459-466

83 Bates NA, Ford KR, Myer GD, Hewett TE. Timing differences in the generation of ground reaction forces between the initial and secondary landing phases of the drop vertical jump. Clin Biomech (Bristol, Avon) 2013;28(7):796-799

84 Bates NA, Ford KR, Myer GD, Hewett TE. Impact differences in ground reaction force and center of mass between the first and second landing phases of a drop vertical jump and their implications for injury risk assessment. J Biomech 2013;46(7):1237-1241

85 Ford KR, Myer GD, Hewett TE. Reliability of landing 3D motion analysis: implications for longitudinal analyses. Med Sci Sports Exerc 2007;39(11):2021-2028

86 Andrews JR, Axe MJ. The classification of knee ligament instability. Orthop Clin North Am 1985;16(1):69-82

87 Lloyd DG, Besier TF. An EMG-driven musculoskeletal model to estimate muscle forces and knee joint moments in vivo. J Biomech 2003;36(6):765-776

88 McLean SG, Huang X, Su A, Van Den Bogert AJ. Sagittal plane biomechanics cannot injure the ACL during sidestep cutting. Clin Biomech (Bristol, Avon) 2004;19(8):828-838

89 Hewett TE, Myer GD, Ford KR. Decrease in neuromuscular control about the knee with maturation in female athletes. J Bone Joint Surg Am 2004;86-A(8):1601-1608

90 Hewett TE, Stroupe AL, Nance TA, Noyes FR. Plyometric training in female athletes. Decreased impact forces and increased hamstring torques. Am J Sports Med 1996;24(6):765-773

91 Kiapour AM, Wordeman SC, Paterno MV, et al. Diagnostic value of knee arthrometry in the prediction of anterior cruciate ligament strain during landing. Am J Sports Med 2014;42(2):312-319

92 Wordeman SC, Paterno MV, Quatman CE, Bates NA, Hewett TE. Arthrometric curve-shape variables to assess anterior cruciate ligament deficiency. Clin Biomech (Bristol, Avon) 2012;27(8): 830-836

93 Tate JJ, Milner CE. Real-time kinematic, temporospatial, and kinetic biofeedback during gait retraining in patients: a systematic review. Phys Ther 2010;90(8):1123-1134

94 Onate JA, Guskiewicz KM, Sullivan RJ. Augmented feedback reduces jump landing forces. J Orthop Sports Phys Ther 2001;31(9): 511-517

95 Verhoeff LL, Horlings CG, Janssen LJ, Bridenbaugh SA, Allum JH. Effects of biofeedback on trunk sway during dual tasking in the healthy young and elderly. Gait Posture 2009;30(1):76-81

96 Teran-Yengle P, Birkhofer R, Weber MA, Patton K, Thatcher E, Yack $\mathrm{HJ}$. Efficacy of gait training with real-time biofeedback in correcting knee hyperextension patterns in young women. J Orthop Sports Phys Ther 2011;41(12):948-952

97 Ford KR, DiCesare CA, Myer GD, Hewett TE. Real-time biofeedback to target risk of anterior cruciate ligament injury: a technical report for injury prevention and rehabilitation. J Sport Rehabil 2015;Technical Notes. doi: 10.1123/jsr.2013-0138 